\title{
El español como lengua extranjera: perspectivas, retos y oportunidades. Una visión panorámica*
}

\author{
Nancy Agray Vargas ${ }^{1}$ \\ Pontificia Universidad Javeriana
}

\section{Resumen}

Este artículo tiene como objetivo presentar una visión panorámica y bastante general de algunas de las razones por las cuales el español como lengua extranjera (ELE) se encuentra en auge en algunos de los contextos que de manera directa afectan al país. Posteriormente, se proponen las perspectivas, retos y oportunidades que el tema representa para las instituciones colombianas dedicadas al tema, y por último, se mencionan los principios que deberían regir las acciones que se emprendan colectivamente para la construcción de la comunidad académica interesada en el español como lengua extranjera.

\section{Abstract}

The main aim of this article is to show a wide and more general scope about some of the reasons why Spanish as a foreign language is on the peak in some of the context that directly affect the country. Afterwards the perspectives, challenges and opportunities this topic brings to the Colombian institutions working on this field are proposed; and finally it is mentioned the principles under which the actions to build academic community interested in Spanish as foreign language should be ruled

\footnotetext{
* Texto escrito de la ponencia presentada en el Primer Encuentro Regional de Español como Lengua Extranjera. Pontificia Universidad Javeriana. Agosto de 2007. 1 Docente-investigadora de la Pontificia Universidad Javeriana. Licenciada en Filología e Idiomas español-inglés, Universidad Nacional de Colombia. Máster en Enseñanza del Español como Lengua Extranjera, Universidad Antonio de Nebrija. Especialista en Estudios Culturales, Pontificia Universidad Javeriana. Miembro de los grupos de investigación: Estudios del Discurso, y Lenguaje y Pedagogía Crítica. Miembro del Consejo Académico del Sistema Internacional de Certificación del Español como Lengua Extranjera SICELE y miembro fundador de la Red Académica para el Desarrollo del Español como Lengua Extranjera EnRedELE. Correo-e: nagray@javeriana.edu.co.
} 
Palabras clave: Español Lengua Extranjera, ELE, perspectivas, retos, oportunidades.
Key words: Spanish as a Foreign Language, SFL, perspectives, challenges, opportunities.

\section{Introducción}

El español como lengua extranjera -ELE- ha venido en un proceso de consolidación como una de las lenguas más habladas en el mundo y como uno de los instrumentos indispensables en la comunicación intercultural y económica en contextos tan diversos como Estados Unidos, Australia, China, Japón, Corea o Canadá, que la ubica actualmente en un lugar preponderante a nivel mundial. Este auge proviene de una serie de factores que conforman un panorama complejo para el análisis, sin embargo, se pueden identificar algunas de las razones que parece tienen más importancia en la producción de este fenómeno. El análisis de la nueva circunstancia del español como lengua extranjera en el mundo permite proponer las que considero algunas perspectivas, retos y oportunidades que tenemos las instituciones interesadas en ELE en el país. Podemos decir, en términos pragmáticos, que se trata de plantear las oportunidades que surgen en esta coyuntura y que deberíamos aprovechar en los diferentes niveles que la constituyen, principalmente en el nivel lengua-cultura y en el nivel lengua-relaciones internacionales.

Las condiciones económicas, políticas, sociales y culturales del mundo actual, que se caracteriza por la globalización de todos los espacios de la vida que el mundo capitalista impone, ha concedido mayor relevancia al conocimiento de, al menos, una lengua extranjera, entre ellas el español. Esas condiciones, así como las oportunidades económicas que el mundo hispano ofrece para diferentes países y el establecimiento de nuevas políticas económicas y lingüísticas hacen prever que en este siglo el español llegará a convertirse en la segunda lengua extranjera más hablada del mundo, después del inglés, según datos de la Enciclopedia del español en el mundo (Instituto Cervantes, 2007).

\section{Razones y contextos}

Históricamente el auge y decadencia de las lenguas está asociado al desarrollo de los imperios y al contexto político y económico predominante. Por ello, no podemos hablar de razones de naturaleza única al analizar el caso de ELE, sino más bien de razones de diversa índole que se determinan mutuamente. Mencionaré cuatro razones asociadas a cuatro contextos particulares. 1- El fenómeno migratorio 
de habitantes de los países hispanoamericanos a Estados Unidos. 2El establecimiento de acuerdos económicos internacionales, como es el caso de Brasil. 3- Los intercambios comerciales con países orientales, en particular con China. 4- La industria turística en España y en Latinoamérica.

En relación con la primera, en el siglo XX aumentóla población hispana en Estados Unidos, constituyéndose en más del $9 \%$ de la población total. Es decir, hay entre siete y diez veces más hispanohablantes que hablantes de otras lenguas diferentes del inglés (Cfr., Instituto Cervantes, 2007). Este aumento de la población de origen hispano en Estados Unidos ha obligado al gobierno norteamericano, como producto de la lucha y resistencia de la población hispana inmigrante que cada día se organiza más y reclama sus derechos, a repensar el país y a tratar de establecer políticas educativas y sociales que afronten esta realidad y den una mayor apertura e inclusión de esta población.

Ello explica que la enseñanza de español como lengua extranjera en este país ocupe el primer lugar, superando al francés, al alemán y al italiano, que el número de estudiantes de español duplique al de los estudiantes de francés, que es la segunda lengua extranjera por número de alumnos, y que la industria editorial en materia de textos de español como lengua extranjera sea, junto con la industria editorial española, una de las más importantes del mundo.

La segunda razón nos ubica en Suramérica, y en particular en Brasil. A propósito de la creación del MERCOSUR, y con ello del aumento en las relaciones comerciales y culturales en la región, por una parte, Brasil ha desarrollado políticas lingüísticas para facilitar el intercambio con los países vecinos, y por otra, los demás países suramericanos, especialmente los miembros del MERCOSUR, han establecido programas de intercambio y fortalecimiento de las lenguas portuguesa y española.

Brasil ha decretado el español como la segunda lengua oficial, de enseñanza obligatoria en la educación secundaria, aunque actualmente en calidad de asignatura electiva, con miras a ser de carácter obligatorio en un futuro cercano, contando con su mayor auge en los estados del sur y del sudeste. En la educación superior actualmente el español se está enseñando en más de 50 universidades públicas y privadas, lo que ha evidenciado una de las mayores problemáticas del país, la falta de profesores de ELE. Esta situación ha desatado una avalancha de proyectos, convenios, reuniones e intereses de instituciones de diferente naturaleza 
para "entrar" a un mercado que se asume como económicamente rentable a propósito de necesidades como la mencionada, así como otras asociadas con ella como la falta de programas de pregrado y posgrado, de intercambio de docentes y estudiantes, de diseño de materiales y de diseño de exámenes de proficiencia.

La tercera razón tiene que ver con política económica. El caso más destacado es el posicionamiento de la China, el país más poblado del mundo, como potencia económica mundial en un futuro no muy lejano, que ha llevado a que diferentes países empiecen a visitar China con el ánimo de establecer relaciones comerciales y culturales con este país, que incluyen, por supuesto, la lengua. Pero este interés no es en una sola vía, pues China también vislumbra muchas posibilidades comerciales con todo el mundo, especialmente con América Latina, y por ello está desarrollando programas de divulgación y promoción de su lengua y su cultura, y está estableciendo alianzas estratégicas para tener mayor acercamiento, a su vez, a la lengua y culturas del español. De hecho, en la actualidad se encuentra en discusión y análisis una ley, ya aprobada en primer debate, por la cual se establece la obligatoriedad del español en los centros educativos de ese país.

Por último, se encuentra la industria del turismo. En España el turismo representa una de las mayores bases de su economía y en los últimos tiempos ha estado ligado a la industria de la lengua, y en Hispanoamérica, el interés por la riqueza natural y cultural de los países que la constituyen ha producido en algunos de ellos una consolidación de su industria turísticolingüística como es el caso de Costa Rica, Argentina y México. En Colombia, esta relación turismolengua, empieza a ser explotada, especialmente en la costa Atlántica y en Bogotá. Estas razones, que explican en parte el auge del español, no son exclusivas de estos contextos; se evidencian en otros como Canadá, Australia y los países africanos, a propósito de políticas migratorias, y Japón y Corea, debido a políticas comerciales.

\section{Respuestas desde España e Hispanoamérica}

Este proceso de desarrollo y consolidación del español como lengua extranjera en el mundo ha tenido respuestas de diferente naturaleza. Vale la pena mencionar, por su impacto y rápido crecimiento, la política lingüística difundida por el gobierno español a través del Instituto Cervantes, organismo creado en los años 90 para encargarse de esta labor, y que a la fecha se ha constituido en la Institución con mayor liderazgo en el tema de ELE. Unida a la anterior, otra respuesta significativa para el desarrollo de la lengua española la constituye 
el trabajo que durante más de diez años ha realizado el grupo de lingüistas aplicados y especialistas en pedagogía del Consejo de Europa al desarrollar y promover documentos académicos que han sido parte de importantes políticas lingüísticas, como por ejemplo, el "Marco de Referencia Europeo para la Enseñanza, el Aprendizaje y la Evaluación de las Lenguas Extranjeras".

En el contexto hispanoamericano las respuestas han sido más aisladas, de carácter local e institucional. México, Argentina y Costa Rica, por ejemplo, son los países donde más consolidada se encuentra la enseñanza del español como lengua extranjera. En el caso de México, debido a la cercanía con Estados Unidos y el menor costo de sus cursos, atrae gran número de estudiantes. En Argentina, su cercanía con Brasil le ha facilitado el desarrollo de convenios, y Costa Rica, gracias a su gran riqueza natural, se ha posicionado como destino turístico-lingüístico. El desarrollo de ELE tanto en México como en Argentina ha significado, por ejemplo, que a la fecha sean los dos únicos países hispanoamericanos con exámenes internacionales para evaluar la proficiencia en español como lengua extranjera.

Países centroamericanos como Cuba, Puerto Rico y República Dominicana, aunque tienen mucho potencial en el campo, por el turismo que atraen, no han logrado mayores desarrollos, dadas sus condiciones económicas y políticas. Actualmente se adelantan conversaciones con miras a la firma de acuerdos entre ellos para desarrollar el tema en el Caribe. En el caso de Uruguay y Paraguay, su participación en el MERCOSUR ha posibilitado el desarrollo de proyectos conjuntos como, por ejemplo, el que están realizando con Brasil, para el diseño, pilotaje e implementación de un examen internacional deELE. Porúltimo, en países como Perú, Ecuador, Bolivia y Venezuela, aunque existen programas de ELE, los desarrollos son incipientes. Sobresale la Universidad peruana Ricardo Palma que, mediante convenios, adelanta programas cortos, no conducentes a título, de formación de profesores chinos.

\section{Perspectivas, retos y oportunidades}

La descripción anterior nos permite identificar algunas de las perspectivas, retosyoportunidades quelas instituciones colombianas tenemos en el tema del español como lengua extranjera. Existen tres acuerdos que nos presentan las mayores perspectivas. Por una parte, la participación de 30 universidades colombianas en el Sistema Internacional de Certificación de Español como Lengua Extranjera, SICELE; por otra, el convenio de cooperación binacional firmado con Brasil, y por último, el convenio de cooperación firmado por el 
gobierno colombiano con China. Los dos primeros fueron firmados en marzo y abril de este año, y el último en el 2005.

ElSICELE es un convenio liderado por el Instituto Cervantes en España e Hispanoamérica y en Colombia por la Asociación Colombiana de Universidades -ASCUN- desde el año 2005. Fue firmado este año en Medellín por más de 60 instituciones hispanoamericanas, entre las que se encuentran como miembros fundadores siete universidades colombianas: la Universidad Nacional, la Universidad del Valle, la Universidad del Norte, la Universidad de Antioquia, la Universidad Autónoma de Bucaramanga, la Universidad Pontificia Bolivariana y la Pontificia Universidad Javeriana. Tiene como objetivo central el desarrollo del español como lengua extranjera, específicamente en lo relacionado con los procesos inherentes a la evaluación. Busca la armonización y establecimiento de estándaresinternacionales para el diseño, pilotaje, aplicación y evaluación de exámenes de proficiencia a nivel mundial y promover el reconocimiento internacional de las certificaciones expedidas por las instituciones integrantes.

El segundo, el convenio binacional con Brasil, que abarca diferentes áreas, incluye como uno de sus objetivos el fomento de las lenguas española y portuguesa. En este convenio es poco lo que se ha avanzado; las únicas actividades realizadas son el levantamiento de una base de datos conjunta, y la inclusión en el portal del Ministerio de Educación, Colombia Aprende, de los programas de ELE que se desarrollan en el país. Por último, el convenio de cooperación colombo china ya ha tenido en el tema de ELE uno de sus primeros frutos: el programa de becas completas ofrecidas por el gobierno colombiano mediante el cual vinieron a diferentes universidades del país ciudadanos chinos a estudiar español. Desafortunadamente, no se conoce la evaluación de este programa ni los resultados de esta experiencia para las instituciones colombianas que acogieron a los estudiantes chinos.

Como se mencionó antes, estos convenios presentan grandes perspectivas de desarrollo para las instituciones colombianas, brindándoles oportunidades en los diferentes ámbitos de ELE como son la formación de profesores, el diseño de materiales y pruebas, el desa-rrollo de investigación pertinente en temas específicos y la participación en el diseño de políticas lingüísticas, entre otras. Sin embargo, estas oportunidades para ser aprovechadas adecuadamente demandan de las instituciones colombianas y de los profesores de ELE asumir varios retos entre los que se pueden mencionar: 
- Eldesarrollodepolíticasinstitucionalesqueposibilitenlacreación de redes, convenios y proyectos de investigación conjuntos y el desarrollo de planes de creación o de fortalecimiento de los programas de ELE. Es indispensable el compromiso institucional y la concientización de las autoridades con respecto a lo que significa este ámbito, de manera que el tema de ELE deje el lugar marginado que aún ocupa en algunas instituciones y se convierta en preocupación de todos los que deben estar involucrados.

- El fortalecimiento de la red nacional de ELE que congregue a las instituciones y docentes dedicados a su enseñanza. Este reto lo hemos asumido, en parte, con el trabajo que hace más de dos años iniciamos los representantes de las siete universidades miembros del SICELE, y hace un año las instituciones miembros del nodo Bogotá del SICELE, producto del cual nació la Red Académica para el Desarrollo del Español como Lengua Extranjera, EnRedELE, Red que organizó el Primer Encuentro Regional de ELE que se realizó en Colombia y que celebró, en agosto de 2008, el segundo encuentro de carácter nacional e internacional.

- La consolidación y difusión del mapa general de la situación del español comolengua extranjera en el país. Trabajo que se ha venido adelantando en los espacios académicos mencionados, pero, que aún no hemos concretado en un instrumento de divulgación.

- La construcción de una agenda nacional alrededor del tema de ELE que contemple la participación como país en los espacios que los convenios firmados nos posibilitan y en otros que consideremos relevantes. El abordaje de un tema amplio y complejo acerca del cual es poco lo que se ha trabajado: el del español como segunda lengua, habida cuenta de la existencia de más de 65 comunidades indígenas en el país para las cuales el español no es su lengua materna.

- Por último, el reto que me parece es al que nos debería conducir todo lo anterior: consolidarnos como una comunidad académica capaz de incidir de manera directa, crítica y reflexiva en el planteamiento de las políticas lingüísticas de español como lengua extranjera tanto en el país como en ámbitos internacionales.

Creo que lo construido hasta ahora por las instituciones de manera individual como por los colectivos académicos mencionados, es significativo; sin embargo, aún falta mucho camino por recorrer, y en ese recorrido esperamos contar con la participación de todas 
aquellas instituciones y docentes que consideren, como nosotros, que éste es un compromiso ético y profesional que nos compete. Este compromiso me parece debe ser asumido teniendo como principios el respeto por la diversidad lingüística y cultural, por las variedades de lengua, por los hablantes y por la profesión docente.

\section{Bibliografía}

El Tiempo. (2005-2007). Notas de prensa. Recuperadas entre Septiembre de 2006 y Mayo de 2007. Disponible en http://www.eltiempo.com/. Instituto Cervantes. (2007). Enciclopedia del español en el mundo. Recuperado en mayo de 2007. Disponible en http://www.cervantes.es

Ministerio de Educación de Brasil. (2007). Recuperado en mayo de 2007. Disponible en http://portal.mec.gov.br/index.php.

Rodríguez, Claudia. (2005). Cursos de español como segunda lengua en universidades latinoamericanas. Informe presentado a la Facultad de Comunicación y Lenguaje de la Pontificia Universidad Javeriana. Bogotá, Colombia. Manuscrito no publicado.

SICELE. (2005-2007). Documentos de trabajo. 